\title{
Selection of Filtration Methods in the Analysis of Motion of Automated Guided Vehicle
}

\author{
Dobrzańska Magdalena $^{1}$, Dobrzański Paweł ${ }^{1}$, Śmieszek Mirosław ${ }^{1}$, Pawlus Paweł ${ }^{2}$ \\ ${ }^{1}$ Faculty of Management, Rzeszów University of Technology, Al. Powstańców Warszawy 10, 35-959 Rzeszów, Poland, \\ e-mail: pd@prz.edu.pl \\ ${ }^{2}$ Faculty of Mechanical Engineering and Aeronautics, Rzeszów University of Technology, Al. Powstańców Warszawy 8 , \\ 35-959 Rzeszów, Poland
}

In this article the issues related to mapping the route and error correction in automated guided vehicle (AGV) movement have been discussed. The nature and size of disruption have been determined using the registered runs in experimental studies. On the basis of the analysis a number of numerical runs have been generated, which mapped possible to obtain runs in a real movement of the vehicle. The obtained data set has been used for further research. The aim of this paper was to test the selected methods of digital filtering on the same data set and determine their effectiveness. The results of simulation studies have been presented in the article. The effectiveness of various methods has been determined and on this basis the conclusions have been drawn.

Keywords: Filtration, Gaussian regression filter, spline filter, automated guided vehicle, position error, odometry.

\section{INTRODUCTION}

For the conduction and determination of the current position of an automated guided vehicle a variety of navigation systems are used to move from the starting point along a specified route to the destination. The basis for determining the current position in the majority of cases is odometry - computational navigation. This method of determining a vehicle's position is laden with many errors [8]-[11], [14], [16] and requires correction [17]. In the process of correction, a range of measurement methods is used. Measuring is followed by correction of the course of the vehicle. Adjustments can be implemented continually or cyclically, after a specified section or passing a specified marker - reference point [1], [4], [7], [13].

The results of the measurements are burdened with distortions; therefore, they must be filtered [17]. Currently, there are many techniques for data filtering. Each of the existing filter algorithms has properties that narrow down the area of their application.

To examine and determine the effectiveness of the filtration methods concerned and their algorithms this paper analyzes numerically generated runs to reflect actual trajectories of an automated guided vehicle. One of the main errors of a navigation system such as odometry is the adoption of an improper ratio between the radii of the left wheel to the right wheel. This error belongs to the group of systematic biases and makes the vehicle stray from the prescribed direction of the route and make a curved motion in an arc of constant radius [2], [15]. The actual runs measured are characterized by the presence of additional oscillation arising from the control system, and the disruption and random error of measurement resulting from the measurement sensors.

The objective of the simulation tests was the choice of the appropriate method of filtration which eliminates this adverse phenomenon, and enables the designation of the radius of the arc which the vehicle moves in. This in turn will allow the designation of improvements to the computational navigation algorithm and making of ongoing course correction.

\section{SUBJECT \& METHODS}

\section{A. Odometry}

The basic method of calculating the position of automated guided vehicle is computational navigation - odometry. It involves determining the current position of the vehicle on the basis of the distance traveled by the marked point on the vehicle $K$. Computational navigation uses the difference in speed between the drive wheels $v_{L}$ and $v_{R}$ to determine the directional angle $\theta$. The nature of this solution is shown in Fig.1. 


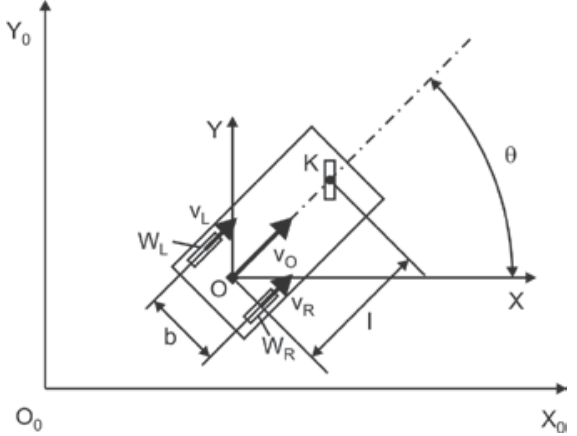

Fig.1. Coordinate system used in analytical navigation.

This method is a continuous count of the distance traveled by the left wheel $\left(W_{L}\right)$ and right wheel $\left(W_{R}\right)$, and the determination in each iteration of the directional angle changes with the motion of the vehicle $\theta$. This is applied in vehicles in which two independently powered drive wheels are used. Appropriate differentiation of the speeds of these wheels forces rotation of the vehicle around a vertical axis of rotation passing through point $O$ and changes in the directional angle $\theta$.

If the position of a selected point $O$ directed using two independently powered wheels $W_{L}$ and $W_{R}$ in the base reference system $X_{0} O_{0} Y_{0}$ (Fig.1.) in iteration $k$ is defined by the state vector $(x(k), y(k), \theta(k))$, the position of the vehicle in the iteration $k+1$ is expressed by:

$$
\left[\begin{array}{l}
x(k+1) \\
y(k+1) \\
\theta(k+1)
\end{array}\right]=\left[\begin{array}{l}
x(k) \\
y(k) \\
\theta(k)
\end{array}\right]+\left[\begin{array}{c}
\Delta t \cdot v_{\mathrm{O}}(k+1) \cdot \cos (\theta(k)+\Delta t \cdot \omega(k+1)) \\
\Delta t \cdot v_{\mathrm{O}}(k+1) \cdot \sin (\theta(k)+\Delta t \cdot \omega(k+1)) \\
\Delta t \cdot \omega(k+1)
\end{array}\right]
$$

Velocities $v_{\mathrm{O}}(k+1)$ and $\omega(k+1)$ can be determined from the following relations:

$$
\begin{gathered}
v_{\mathbf{O}}(k+1)=\left(v_{R}(k+1)+v_{L}(k+1)\right) / 2 \\
\omega(k+1)=\left(v_{R}(k+1)-v_{L}(k+1)\right) / b
\end{gathered}
$$

where:

$v_{R}(k+1)$ - speed of the right wheel,

$v_{L}(k+1)$ - speed of the left wheel,

$b$ - wheelbase of the driven wheels.

Velocity $v_{R}(k+1)$ and $v_{L}(k+1)$ can be determined from the following relations:

$$
\begin{aligned}
& v_{R}(k+1)=\omega_{R}(k+1) \cdot r \\
& v_{L}(k+1)=\omega_{L}(k+1) \cdot r
\end{aligned}
$$

where:

$r$ - radius of the steered wheels.

In the above consideration it was assumed that the wheels are rigid and they roll without spin, the contact between the wheel and the floor is a point contact and the radii $r$ of the driven wheels are the same.

Presented method is very simple but has got some drawbacks connected with errors. We can distinguish several sources of errors that have an impact on the accuracy of positioning. These sources were divided into two categories: systematic errors and non-systematic errors. Additional odometry errors can be caused by the odometry equations themselves, since they approximate an arbitrary motion as a series of short rectilinear segments. The precision of this approximation depends on the program step.

Two dominant sources of errors in odometry are unequal wheel diameters and uncertainty about the wheelbase.

By properly determined and set radii $r_{L}$ and $r_{P}$ a vehicle carries out straight motion. In the event of a change of these radii, for example, as a result of loading of the vehicle with transported cargo, the vehicle carries out a curvilinear motion. An example run registered during the motion using laser rangefinders is shown in Fig.2. [17]. The determination on a straight test section of radial motion on the curvature of the course will make it possible to determine amendments to the calculated algorithm for the correction of the course. Measuring errors also overlap with the measurements and the shape of the trajectory, which is well illustrated in Fig.2. showing the actual run.

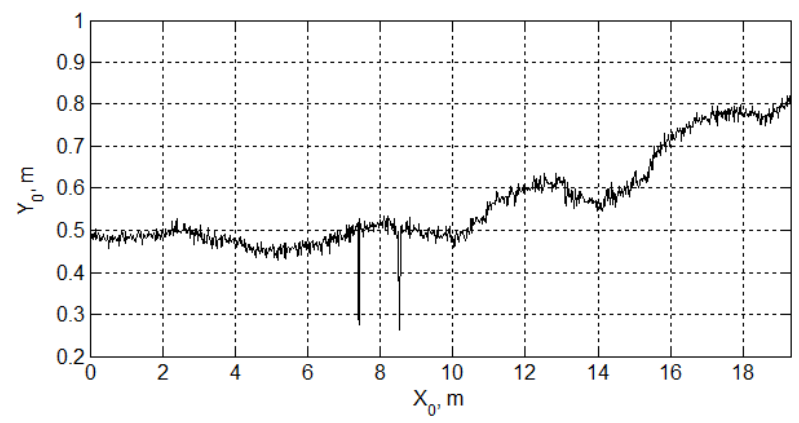

Fig.2. The real run of automated guided vehicle.

The registered run is curved. We can distinguish an arc of large radius, oscillations on the arc and measuring errors in the form of noise. The arc of large radius is a result of the adoption of an improper ratio of the radius of the left wheel to the right wheel. Meanwhile, the presence of oscillation results from the regulation set-up used.

Assuming various errors in relation to the radii of rolling wheels, numerical runs were generated to reflect sample trajectories of an automatically driven transport vehicle. Fig.3.a) shows a sample numerically generated run and its components in the form of an arc (Fig.3.b)), its oscillation (Fig.3.c)), and measurement noise (Fig.3.d)).

Since the purpose of simulation tests was to find the most appropriate method of filtration in a group of numerically generated runs in accordance with the provisions shown in Fig.3., it was decided to examine the effectiveness of the methods of filtration considered in the latter part of this article. 

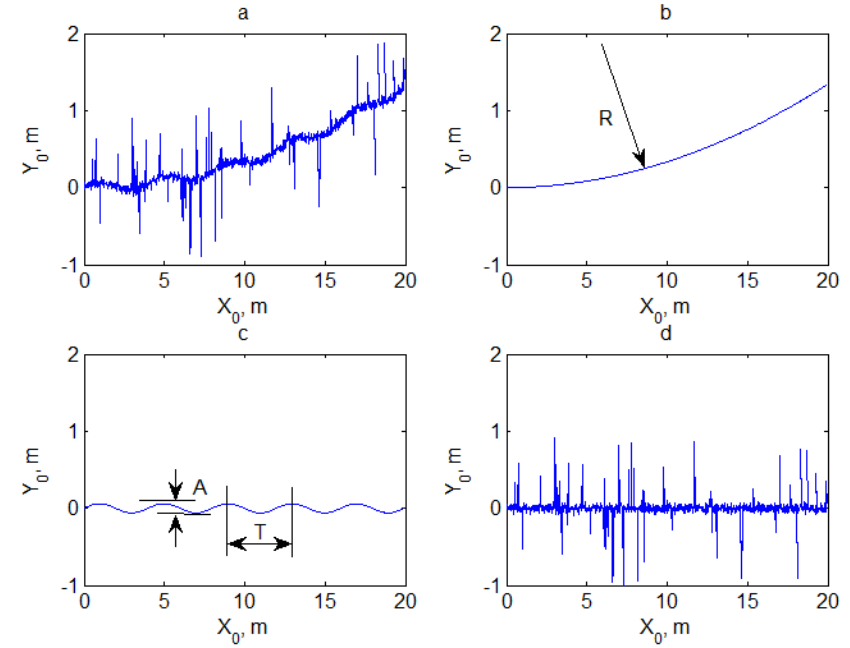

Fig.3. The numerically generated run reflects the exemplary trajectory of automated guided vehicle.

\section{B. Filtration methods}

In the simulation studies, it was decided to examine the effectiveness of Gaussian regression filters of zero and second order, spline filters, and robust regression filters.

The designation of the middle line of the Gaussian regression filter $w(x)$ of zero order is done using regression. The advantage of this filter is that for each point of the measured section the value of the mean line of the filter is defined. The regression arrangement can be mathematically described by the following relationship [3]:

$$
\int_{x_{1}}^{x_{2}}(z(x-\xi)-w(x))^{2} \cdot s(\xi) \cdot d \xi \rightarrow \min _{w(x)}
$$

The value of the mean line of the filter $w(x)$ of the filtered profile reduces the squared deviation of the measured profile $z(x-\xi)$ weighted by $s(\xi)$ and integrated in the range of $0<(x-\xi)<l$ ( $l-$ measured length). The limits of integration are defined in such a way that guarantees analysis of the entire section measured.

The mean line of the filter $(x)$ is described by the following formula:

$$
w(x)=\int_{x_{1}}^{x_{2}} z(x-\xi) \cdot s_{0}(\xi) \cdot d \xi
$$

Where

$$
s_{0}(\xi)=s(\xi) / \int_{x_{1}}^{x_{2}} s(\xi) \cdot d \xi
$$

In addition, the regression filter performs a weighted averaging (shown in equations 7 and 8). Averaging occurs in a finite interval. The weight function $s_{0}(x)$ is scalable, so its area is always set to 1 . The regression filter corresponds to the filtration with phase correction in accordance with ISO 11562 standard, when the weight of $s(x)$ is replaced with a Gaussian probability function and the limits of integration are in the range $-\infty<x-\xi<\infty$. The Gaussian regression filter allows the avoidance of the effects of the final profile by changing the weight function for the initial and final parts.

Gaussian regression filter of second order can approximate distinct form components since it fits a polynomial curve locally.

The robust filter is a modification of the Gaussian filter involving the introduction of an additional weight function:

$$
w(x)=\int_{-\infty}^{\infty} z(x-\xi) \cdot \rho(x) s(\xi) \cdot d \xi
$$

The additional function of the weight decreases in abnormal places deviating far from the mean.

The robust filter also responds well to the peaks of the profiles. This filter applied to the profiles not containing outliers should have the characteristics of a Gaussian filter.

Spline filter is a special type of discrete linear filter. Spline filters are characterized by the spline function to obtain a filtered output signal. The function of $S(x)=S\left(x, \Delta_{n}\right)$ defined on the interval $\langle a, b\rangle$ is called the function splined to the degree of $m(m \geq 1)$ if:

1. $S(x)$ is a polynomial of degree at most $m$ in each interval $\left(x_{i}, x_{i+1}\right), i=0,1, \ldots, n-1$.

2. $S(x)$ and its derivative step $1,2, \ldots, m-1$ are continuous in the intervals.

Any kind of function very often comes close to spline functions. This is easily linked to the designation of their values and convergence for the numerous classes of functions. In practice, functions splined to a third degree (cubic) are often used, which for many issues are sufficiently smooth and the speed of their convergence is satisfactory.

The current study demonstrated the flexibility and usability of spline filters for industrial applications.

Filters based on spline curves are based on natural cubic spline functions.

The weight function of this type of spline filters cannot be specified unambiguously. Therefore, filter equations are used instead of weight functions to describe the spline filter. However, the numerical calculation of the weight function for spline filters is always possible, when necessary. If the sampling interval is small enough and the spline filter is based on the cubic curve, weight can be approximated by a continuous function [12]:

$$
s(x)=\frac{\pi}{\lambda_{c}} \sin \left(\sqrt{2} \frac{\pi}{\lambda_{c}}|x|+\frac{\pi}{4}\right) \exp \left(\sqrt{2} \frac{\pi}{\lambda_{c}}|x|\right)
$$


Filter equations are based on cubic splines and have the following form:

$$
\left(1+\alpha^{4} Q\right) w=z
$$

where $Q$ is the $n$ dimensional matrix.

In a non-periodical case:

$$
Q=\left(\begin{array}{ccccccc}
1 & -2 & 1 & & & & \\
-2 & 5 & -4 & 1 & & & \\
1 & -4 & 6 & -4 & 1 & & \\
& \ddots & \ddots & \ddots & \ddots & \ddots & \\
& & 1 & -4 & 6 & -4 & 1 \\
& & & 1 & -4 & 5 & -2 \\
& & & & 1 & -2 & 1
\end{array}\right)
$$

In a periodical case:

$$
Q=\left(\begin{array}{ccccccc}
6 & -4 & 1 & & & 1 & -4 \\
-4 & 6 & -4 & 1 & & & 1 \\
1 & -4 & 6 & -4 & 1 & & \\
& \ddots & \ddots & \ddots & \ddots & \ddots & \\
& & 1 & -4 & 6 & -4 & 1 \\
1 & & & 1 & -4 & 6 & -4 \\
-4 & 1 & & & 1 & -4 & 6
\end{array}\right)
$$

However, the parameter $\alpha$ is expressed by the formula:

$$
\alpha=\frac{1}{2 \sin \frac{\pi \Delta x}{\lambda_{\mathrm{CO}}}}
$$

where:

$n$ - number of measured points in the profile,

$z$ - vector with a dimension of $n$ that contains the coordinates of the profile before filtration,

$w$ - vector with levels after filtration, dimension of $n$ that contains the levels after filtration,

$\lambda_{C O}$ - wavelength limit of filtered profile,

$\Delta x$ - sampling interval.

Filters described above are widely used in surface metrology; however, areas of their application are wider [5], [6].

\section{SimULATION STUDIES}

\section{A. Description of tests}

The simulation studies carried out consisted of two stages. In the first stage, the maximum permissible errors are specified to ensure a collision-free passage on a given stretch of the route with an arc of specified radius. This stage includes the different values of the arcs and the corresponding allowable measurement errors. In the second phase the results of simulation studies were presented in which trajectory runs were generated and different methods of filtration were used.

B. Determination of the maximum permissible errors
For the determination of the maximum permissible errors in the study a case was considered of an AGV vehicle with dimensions $0.8 \times 1.2 \mathrm{~m}$ in a corridor of length $s=20 \mathrm{~m}$ and the final part of its journey through a gate with a width of $1.2 \mathrm{~m}$. In real conditions the radii of the wheels change, e.g. as a result of the load of transported cargo on the vehicle. Therefore, the vehicle carries out a curvilinear motion (Fig.2.). The measurement system and calculation algorithm applied in the vehicle allow to make a course correction in order to implement direct motion along the axis of the corridor. The estimated radius of the trajectory differs from the real value and is burdened with some error. Wherein, the estimated value may be higher or lower than the actual value. The determined corrections to the motion control algorithm do not allow to maintain a straight trajectory. In order to determine the limits of errors to ensure free passage for a predetermined corridor, there were specified permissible errors in determining radii of curvilinear motion.

Simulation studies have been considered with curvature of the track of 50,100,150, 200, 250, and $300 \mathrm{~m}$. The results of the respective simulation are shown in Fig.4.

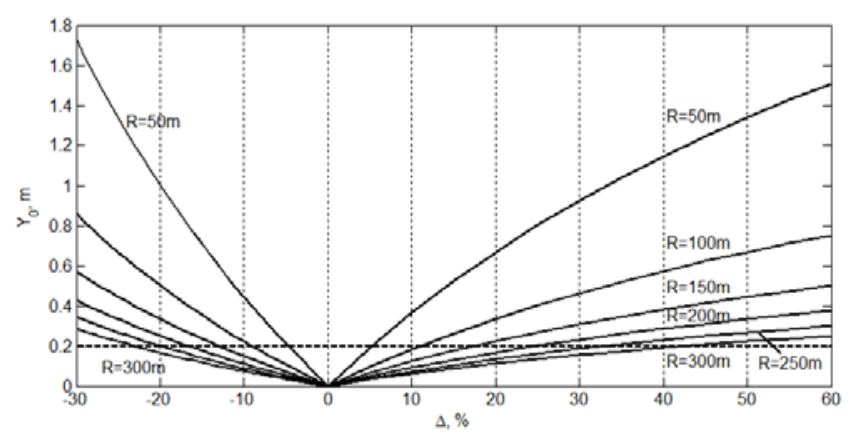

Fig.4. Errors of radii determination and corresponding deviations from assumed tracks.

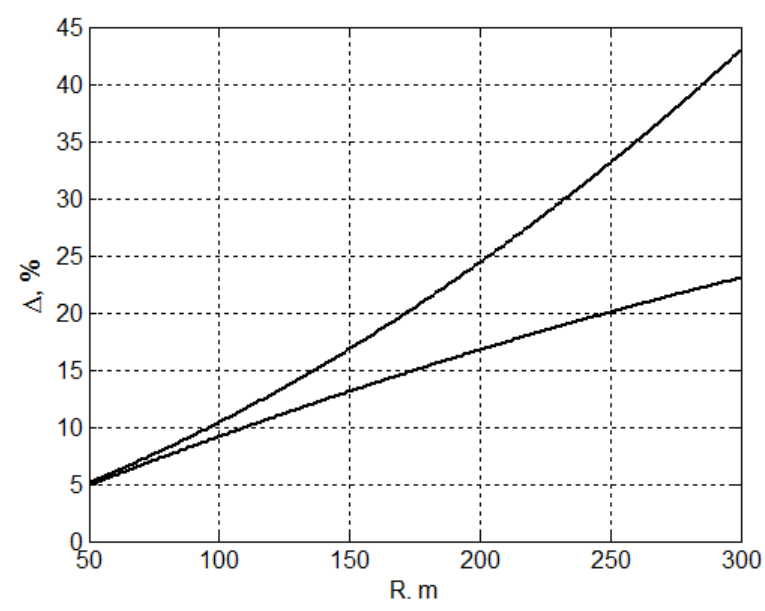

Fig.5. Permissible error values.

The curves shown in Fig.4., diverging from the point 0, show the deviations from the target track at a $20 \mathrm{~m}$ distance 
of motion for different basic radii $\mathrm{R}$ in the error estimation function. The estimation error $\Delta$ has a value greater than zero when the real radius is smaller than the estimated value. Otherwise, we are dealing with a negative error. The horizontal line in Fig.4. determines the maximum permissible deviation from the target trajectory. In the considerations it has been assumed that the deviation is $0.2 \mathrm{~m}$. The values of deviations from a given course, for the same basic radii $\mathrm{R}$ have different courses for positive and negative errors. In order to demonstrate the differences, the graph in Fig.5. has been developed.

The vertical axis defines the maximum value of error estimated for the larger radii than the basic one (bottom curve), and the absolute values of the errors for the radii smaller than the basic one (upper curve).

\section{Filtrations and generations of radii}

In simulation, computer generated runs corresponding to trajectories of automated guide vehicles were analyzed. These trajectories are subjected to various disturbances mentioned above. Effectiveness of selected methods of digital filtering was analyzed. Regression filters of zero (G0) and second order (G2) as well as robust (RO) and spline filters (SP) were studied. It was assumed that the analyzed profiles are characterized by radii (see Fig.3.) of 50, 100, 150, 200, 250, and $300 \mathrm{~m}$, respectively. Fig.6. presents an example of profile of $20 \mathrm{~m}$ length. The results of filtration using the selected filter $s$ (mean lines) are also shown. Simulation analysis was done for profiles of amplitude $\mathrm{A}=$ $0.05 \mathrm{~m}$ and wavelength $T=4 \mathrm{~m}$ (Fig.3.). The cut-off wavelength changed from 0.4 to $0.9 \mathrm{~m}$ with interval of $0.1 \mathrm{~m}$.

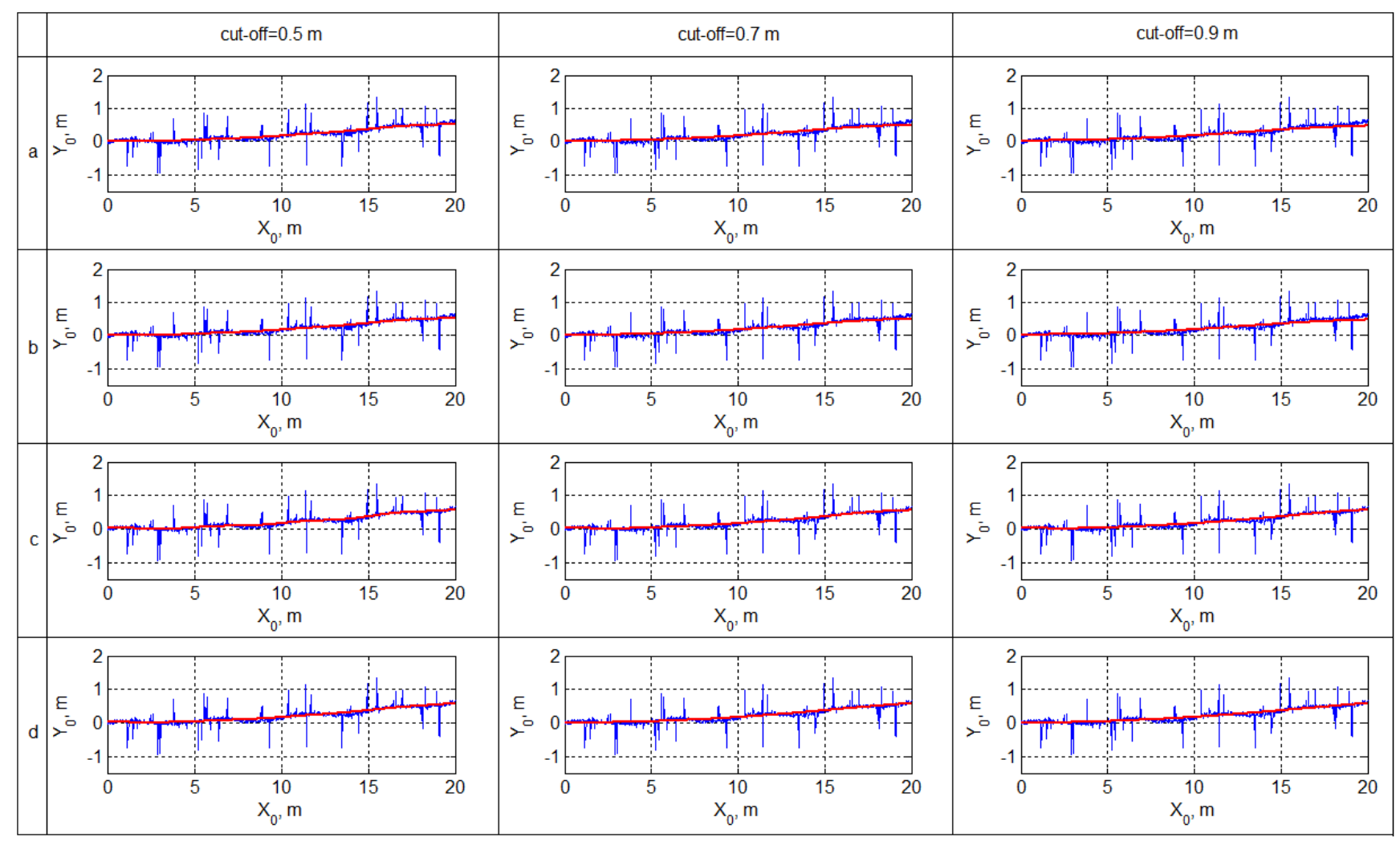

Fig.6. Results of filtration for profile of the base radius $R=250 \mathrm{~m}$.

\section{RESULTS AND DISCUSSION}

Application of filters commonly used in surface metrology to navigation problem is novelty of this research. In order to assess behaviors of selected filters, difference between assumed base radius and radius after filtering was assumed as measure of filter effectiveness. The relative error $\Delta$ was calculated as ratio (R-F)/R, where $\mathrm{R}$ is assumed base radius and $\mathrm{F}$ is radius after filtration. In all the presented series of results the following succession of filters: robust, regression with zero order, regression with second order, and spline filter was used.

Table 1. - Table 6. present relative errors for various base radii after application of various filters with different cutoffs. On the basis of results shown in Fig.5. the maximum errors of radius of curvature matching were calculated for the base radii of $50,100,150,200,250,300 \mathrm{~m}$. They are $5 \%, 9 \%, 13 \%$, $17 \%$, $20 \%$, $23 \%$, respectively. When obtained deviations are smaller than limiting errors, the cells in Tables are marked in yellow. 
Table 1. Relative error $\Delta$ for the base radius $R=50 \mathrm{~m}$.

\begin{tabular}{|c|c|c|c|c|c|}
\hline \multicolumn{7}{|c|}{$R=50 \mathrm{~m}$} \\
\hline Lp. & cut-off, m & GO $\Delta, \%$ & R0 $\Delta, \%$ & G2 $\Delta, \%$ & SP $\Delta, \%$ \\
\hline 1 & 0.4 & 16.0617 & 16.0798 & 18.9404 & 18.4932 \\
\hline 2 & 0.5 & 13.7798 & 13.7842 & 18.7525 & 18.3961 \\
\hline 3 & 0.6 & 11.0780 & 11.0724 & 18.5901 & 18.3596 \\
\hline 4 & 0.7 & 7.9976 & 7.9839 & 18.4810 & 18.3632 \\
\hline 5 & 0.8 & 4.5378 & 4.5171 & 18.4021 & 18.3872 \\
\hline 6 & 0.9 & 0.6793 & 0.6519 & 18.3425 & 18.4185 \\
\hline
\end{tabular}

Table 2. Relative error $\Delta$ for the base radius $R=100 \mathrm{~m}$.

\begin{tabular}{|c|c|c|c|c|c|}
\hline \multicolumn{7}{|c|}{$R=100 \mathrm{~m}$} \\
\hline Lp. & cut-off, m & GO $\Delta, \%$ & R0 $\Delta, \%$ & G2 $\Delta, \%$ & SP $\Delta, \%$ \\
\hline 1 & 0.4 & -7.7057 & -7.5965 & -4.4641 & -4.4211 \\
\hline 2 & 0.5 & -10.3958 & -10.3048 & -3.6530 & -4.6373 \\
\hline 3 & 0.6 & -13.9309 & -13.8519 & -3.6659 & -4.7567 \\
\hline 4 & 0.7 & -18.0889 & -18.0189 & -4.0446 & -4.7526 \\
\hline 5 & 0.8 & -22.7811 & -22.7184 & -4.4373 & -4.6803 \\
\hline 6 & 0.9 & -28.0069 & -27.9502 & -4.7340 & -4.5868 \\
\hline
\end{tabular}

Table 3. Relative error $\Delta$ for the base radius $R=150 \mathrm{~m}$.

\begin{tabular}{|c|c|c|c|c|c|}
\hline \multicolumn{7}{|c|}{$R=150 \mathrm{~m}$} \\
\hline Lp. & Cut-off, m & GO $\Delta, \%$ & R0 $\Delta, \%$ & G2 $\Delta, \%$ & SP $\Delta, \%$ \\
\hline 1 & 0.4 & -7.9642 & -7.8311 & -5.4001 & -4.6762 \\
\hline 2 & 0.5 & -10.3022 & -10.1901 & -3.8021 & -4.8310 \\
\hline 3 & 0.6 & -13.6715 & -13.5717 & -3.5715 & -4.9341 \\
\hline 4 & 0.7 & -17.7514 & -17.6603 & -3.9916 & -4.8941 \\
\hline 5 & 0.8 & -22.4008 & -22.3165 & -4.4974 & -4.7715 \\
\hline 6 & 0.9 & -27.5990 & -27.5203 & -4.8955 & -4.6268 \\
\hline
\end{tabular}

Table 4. Relative error $\Delta$ for the base radius $R=200 \mathrm{~m}$.

\begin{tabular}{|c|c|c|c|c|c|}
\hline \multicolumn{7}{|c|}{$R=200 \mathrm{~m}$} \\
\hline Lp. & cut-off, m & GO $\Delta, \%$ & R0 $\Delta, \%$ & G2 $\Delta, \%$ & SP $\Delta, \%$ \\
\hline 1 & 0.4 & -9.3048 & -9.1547 & -7.1044 & -6.0442 \\
\hline 2 & 0.5 & -11.3747 & -11.2479 & -4.8761 & -6.2413 \\
\hline 3 & 0.6 & -14.6456 & -14.5317 & -4.5372 & -6.3771 \\
\hline 4 & 0.7 & -18.7043 & -18.5992 & -5.0954 & -6.3210 \\
\hline 5 & 0.8 & -23.3619 & -23.2638 & -5.7787 & -6.1539 \\
\hline 6 & 0.9 & -28.5825 & -28.4901 & -6.3197 & -5.9581 \\
\hline
\end{tabular}

Table 5. Relative error $\Delta$ for the base radius $R=250 \mathrm{~m}$.

\begin{tabular}{|c|c|c|c|c|c|}
\hline \multicolumn{7}{|c|}{$R=250 \mathrm{~m}$} \\
\hline Lp. & cut-off, m & GO $\Delta, \%$ & R0 $\Delta, \%$ & G2 $\Delta, \%$ & SP $\Delta, \%$ \\
\hline 1 & 0.4 & -10.7725 & -10.6084 & -8.9340 & -7.5491 \\
\hline 2 & 0.5 & -12.5647 & -12.4263 & -6.0555 & -7.8001 \\
\hline 3 & 0.6 & -15.7343 & -15.6096 & -5.6159 & -7.9748 \\
\hline 4 & 0.7 & -19.7703 & -19.6548 & -6.3267 & -7.9034 \\
\hline 5 & 0.8 & -24.4353 & -24.3270 & -7.2019 & -7.6896 \\
\hline 6 & 0.9 & -29.6771 & -29.5746 & -7.8973 & -7.4397 \\
\hline
\end{tabular}

Table 6. Relative error $\Delta$ for the base radius $R=300 \mathrm{~m}$.

\begin{tabular}{|c|c|c|c|c|c|}
\hline \multicolumn{7}{|c|}{$R=300 \mathrm{~m}$} \\
\hline Lp. & cut-off, m & GO $\Delta, \%$ & R0 $\Delta, \%$ & G2 $\Delta, \%$ & SP $\Delta, \%$ \\
\hline 1 & 0.4 & -12.3092 & -12.1323 & -10.8486 & -9.1293 \\
\hline 2 & 0.5 & -13.8099 & -13.6613 & -7.2878 & -9.4405 \\
\hline 3 & 0.6 & -16.8731 & -16.7391 & -6.7471 & -9.6578 \\
\hline 4 & 0.7 & -20.8844 & -20.7600 & -7.6201 & -9.5707 \\
\hline 5 & 0.8 & -25.5551 & -25.4384 & -8.6990 & -9.3076 \\
\hline 6 & 0.9 & -30.8170 & -30.7064 & -9.5590 & -9.0002 \\
\hline
\end{tabular}

Behavior of Gaussian regression filter G0 of zero order was similar to that of robust filter R0, although the analyzed profiles contained outliers (individual peaks of valleys). Presumably the widths of these valleys of peaks were small, therefore robust filter behaved neutrally. The application of robust filter $\mathrm{RO}$ is more time consuming than that of the regression filter G0, therefore, for the analyzed cases the last G0 filter should be preferred. For the smallest radius $\mathrm{R}=50 \mathrm{~m}$ the criterion was fulfilled only after application of robust filter (RO) and regression filter of zero order (G0) for cut-off wavelengths of 0.8 and $0.9 \mathrm{~m}$. The behavior of other filters was worse; deviations were larger than $18 \%$, the largest matching error was found for regression filter of the second order; about 4 times bigger than permissible error. Probably profile with small radius $R$ was similar to surface profile, it contained waviness and roughness (without form) for this profile type, Gaussian filter G0 was better than spline filter SP of not specified weight function. Mean line determined by Gaussian regression filter of second order contained not only waviness but also form. Therefore, behaviors of G2 and SP filters were worse than those of G0 and RO filters

Different situation occurred for higher radii R. In those cases, contrary to the smallest radius $\mathrm{R}$, radii after filtering were larger than assumed. Application of spline filter SP and regression filter of the second order assured correct results for all the cut-offs applied. Only in one case the absolute value of the relative error was higher than $10 \%$ (regression filter of the second order, cut-off $0.4 \mathrm{~m}$ ). The tendency was found that absolute relative errors were higher for larger radius $R$. However, after application of robust filter and regression filter of zero order the errors were higher than after the use of the other filters. Increase of error due to growth of cut-off is the characteristic feature of GO and R0 filters. After application of these filters, the matching criterion was fulfilled only for cut-off of $0.4 \mathrm{~m}$ for radius $R$ of $100 \mathrm{~m}$. For radius $R$ of $150 \mathrm{~m}$ the matching criterion was fulfilled for cut-offs of 0.4 and $0.5 \mathrm{~m}$, for radius $R$ of $200 \mathrm{~m}$ for cut-offs of $0.4,0.5$ and $0.6 \mathrm{~m}$. However, for the largest radii $R$ of 250 and $300 \mathrm{~m} \mathrm{RO}$ and G0 filters behaved correctly for cut-offs between 0.4 and $0.7 \mathrm{~m}$. The absolute value matching error after application of robust and Gaussian regression filter of zero order was up to 31 (for the highest radius $R$ and largest cut-off used). These behaviors of the analyzed filter were caused by the fact that filtered profiles of radii $\mathrm{R}$ higher than $50 \mathrm{~m}$ contained not 
only features similar to roughness and waviness of surface texture, but also form. Gaussian filter of second order G2 reacted correctly since it can approximate form components. It is also known that spline filter SP can remove form.

\section{CONCLUSION}

In order to make corrections in trajectory of vehicle movement the information about course of this trajectory is needed. Determined trajectory from the measurement is subjected to many errors. Therefore, it should be filtered. It was found that filters commonly used in surface texture analysis can be applied in vehicle navigation. Behavior of different filters depends on the trajectory radius. In practical situations movement along a smaller radius is the most dangerous. Deviations from assumed trajectory can be high. For the smallest radius of $50 \mathrm{~m}$ the regression filter of zero order and robust filter were the most effective for cut-offs of 0.8 and $0.9 \mathrm{~m}$. However, for the larger radii of tracks the application of regression filter of the second order and spline filter assured correct results. Therefore, the authors of the present paper recommend selection of the type of filter after initial determination of radii of tracks. For the analyzed trajectories it is not necessary to use robust filter; the Gaussian regression filter is preferred.

\section{REFERENCES}

[1] Bojja, J., Kirkko-Jaakkola, M., Collin, J., Takala, J. (2014). Indoor localization methods using dead reckoning and 3D map matching. Journal of Signal Processing Systems, 76, 301-312.

[2] Borenstein, J., Feng, L. (1996). Measurement and correction of systematic odometry errors in mobile robots. IEEE Transactions on Robotics and Automation, 12 (6), 869-880.

[3] Brinkmann, S., Bodschwinna, H. (2003). Advanced Gaussian filters. In Advanced Techniques for Assessment Surface Topography. ButterworthHeineman, 63-89.

[4] Chen, X., Jia, Y. (2014). Indoor localization for mobile robots using lampshade corners as landmarks: Visual system calibration, feature extraction and experiments. International Journal of Control, Automation, and Systems, 12 (6), 1313-1322.

[5] Dobrzanski, P., Pawlus, P. (2010). Digital filtering of surface topography: Part I. Separation of one-process surface roughness and waviness by Gaussian convolution, Gaussian regression and spline filters. Precision Engineering, 34 (3), 647-650.
[6] Dobrzanski, P., Pawlus, P. (2010). Digital filtering of surface topography: Part II. Applications of robust and valley suppression filters. Precision Engineering, 34 (3), 651-658.

[7] Epton, T., Hoover, A. (2012). Improving odometry using a controlled point laser. Autonomous Robots, 32, 165-172.

[8] Jung, Ch., Moon, Ch., Jung, D., Choi, J., Chung, W. (2014). Design of test track for accurate calibration of two wheel differential mobile robots. International Journal of Precision Engineering and Manufacturing, 15 (1), 53-61.

[9] Kelly, A. (2004). Fast and easy systematic and stochastic odometry calibration. In International Conference on Intelligent Robots and Systems. IEEE, Vol. 4, 3188-3194.

[10] Kelly, A. (2004). Linearized error propagation in odometry. International Journal of Robotics Research, 23 (2), 179-218.

[11] Knuth, J., Barooah, P. (2013). Error growth in position estimation from noisy relative pose measurements. Robotics and Autonomous Systems, 61, 229-244.

[12] Krystek, M. (2000). Discrete linear profile filters. In $X$ International Coloquium on Surfaces, Chemnitz, Germany, 145-152.

[13] Martínez-Barbera, H., Herrero-Pérez, D. (2010). Autonomous navigation of an automated guided vehicle in industrial environments. Robotics and Computer-Integrated Manufacturing, 26, 296-311.

[14] Meng, Q., Bischoff, R. (2005). Odometry based pose determination and errors measurement for a mobile robot with two steerable drive wheels. Journal of Intelligent and Robotic Systems, 41 (4), 263-282.

[15] Muniandya, M., Muthusamyb, M. (2012). An innovative design to improve systematic odometry error in nonholonomic wheeled mobile robots. Procedia Engineering, 41, 436-442.

[16] Ojeda, L., Borenstein, J. (2004). Methods for the reduction of odometry errors in overconstrained mobile robots. Autonomous Robots, 16, 273-286.

[17] Smieszek, M., Dobrzanska, M. (2015). Application of Kalman Filter in navigation process of automated guided vehicles. Metrology and Measurement Systems, 22 (3), 443-454.

Received February 22, 2016. Accepted July 08, 2016. 